Please do not remove this page

RMIT

UNIVERSITY

\title{
Composite video noise modelling and suppressing
}

Xu, Zhengya; Wu, Hong Ren; Yu, Xinghuo; Man, Zhihong; Tan, Damian

https://researchrepository.rmit.edu.au/esploro/outputs/9921858305401341/filesAndLinks?institution=61RMIT_INST\&index=null

Xu, Z., Wu, H. R., Yu, X., Man, Z., \& Tan, D. (2011). Composite video noise modelling and suppressing. Proceedings of the 13th IASTED International Conference on Signal and Image Processing, 43-49. https://doi.org/10.2316/P.2011.759-048

Document Version: Published Version

Published Version: https://doi.org/10.2316/P.2011.759-048

Repository homepage: https://researchrepository.rmit.edu.au

Downloaded On 2023/04/26 19:22:41 +1000

Please do not remove this page 
Thank you for downloading this document from the RMIT Research Repository.

The RMIT Research Repository is an open access database showcasing the research outputs of RMIT University researchers.

RMIT Research Repository: http://researchbank.rmit.edu.au/

\section{Citation:}

Xu, Z, Wu, H, Yu, X, Man, Z and Tan, D 2011, 'Composite video noise modelling and suppressing', in K.R. Rao (ed.) Proceedings of the 13th IASTED International Conference on Signal and Image Processing, Dallas, United States of America, 14-16 December 2011, pp. 43-49.

See this record in the RMIT Research Repository at:

https://researchbank.rmit.edu.au/view/rmit:14021

Version: Published Version

Copyright Statement: (c)

Link to Published Version:

http://dx.doi.org/10.2316/P.2011.759-048 


\title{
COMPOSITE VIDEO NOISE MODELLING AND SUPPRESSING
}

\author{
Zhengya $\mathrm{Xu}^{*}$, Hong Ren $\mathrm{Wu}^{*}$, Xinghuo Yu* Zhihong Man ${ }^{* *}$ and Damian M. Tan ${ }^{*}$ \\ *School of Electrical and Computer Engineering, Platform Technologies Research Institute, RMIT University, Australia \\ ${ }^{* *}$ Faculty of Engineering and Industrial Sciences, Swinburne University of Technology, Australia \\ zhengya.xu@rmit.edu.au; henry.wu@rmit.edu.au; x.yu@rmit.edu.au; zman@swin.edu.au; damian.tan@rmit.edu.au
}

\begin{abstract}
In order to tackle the recording video denoising problem with non-stationary image contents, a critical task is to estimate statistical properties of the composite noise in video signals. After investigation of a variety of test video sequences, based on the wavelet decomposition of the noise, the estimation can be conducted by empirical modeling of the marginal distributions of wavelet coefficients as a subband-dependent parameterized generalized Gaussian distribution. Further development of a new spatio-temporal based composite noise suppression technique is also provided to restore surveillance video, based on the estimation of the parameters of the model. The experiments showed that the proposed techniques obtained promising results.
\end{abstract}

\section{KEY WORDS}

Video processing, Noise modelling, Denoising, Video Quality.

\section{Introduction}

When a video is acquired or converted from one form to another e.g. digitised or compressed, many types of noise or noise-like degradations can be presented in the video. Now, digital video has gradually replaced analogue video in all sectors of electronic visual business/techniques, however, a substantial volume of recorded analogue video material, e.g. surveillance video and archival, remains in existence. These recorded videos are often stored using VHS (Video Home System) or S-VHS (Super-VHS) cassettes with composite video format [1]. When people do image processing using composite videos, they have to deal with the effects of analogue conversion and recording noise.

Usually, solutions of suppress video noise rely on signal modelling and the noise modelling. Generally, noise in images/video is assumed to be additive and randomly distributed with uniform, Gaussian, Laplacian, Negative exponential, Raleigh, or Poisson distributions [2, 3] or modelled as impulse (salt \& pepper or random value) noise [5] or a mixture of both with noise model parameters either known or unknown a priori [4]. The effectiveness of these statistical models has been demonstrated in a range of applications [3]. However, for systems that produce composite noise/structured distortions, e.g., digital coding or form conversions, these statistical noise models may not perform adequately because this sort of noise become some extent correlated to the signals[2, 3]. The modelling of the composite noise is still remained as a challenge problem.

In order to efficiently suppress the noise to enhance the composite video quality for further video processing, the noise/artefacts of the composite video should be isolated (acquired), analysed and modelled. In this paper, since direct statistical modelling of the noise information to capture some common features of the noise is very difficult, first, based on wavelet decomposition approaches, the noise was analysed using a variety of video sequences. Then, a new empirical modeling of the marginal distributions of wavelet coefficients as a subband-dependent mixture of independent, parameterized generalized Gaussian distribution is provided to deal with this challenge problem. After the noise is modelled, the approach to suppress the noise in surveillance videos is proposed based on the model. The experiments were conducted to verify the approach and model. It shows expected results to suppress the noise.

The paper is organized as follows. In Section 2, the composite noise modelling is presented to describe various statistical properties of the noises based on wavelet decomposition after acquisition of the composite video noise. The algorithm to suppress the noise based on the modelling of the noise then is described in Section 3. Experiments to verification of the proposed technique using test video sequences degraded by the composite noises are presented in Section 4. Finally, the conclusion is drawn in Section 5.

\section{Composite noise modelling}

\subsection{Acquisition of the composite noise}

In this paper, let $C_{n} \equiv\left\{\mathbf{c}=\left(c_{1}, c_{2}, n\right) \mid 1 \leq c_{1} \leq H, 1 \leq c_{2} \leq W\right\}$ denote the pixel coordinates of a color frame of a surveillance video, where $H$ and $W$ are the height and the width of the frame, respectively; the subscript $n$ denotes the video frame 
number for $1 \leq n \leq N$. At each pixel coordinate $\mathbf{c} \in C_{n}$, and $V=\left\{C_{n}\right\}_{n=1}^{N}$, where $V$ is a set of pixel positions of a video with $N$ frames, a multivariate value $\mathbf{I}(\mathbf{c})=\left[I_{R}(\mathbf{c}), I_{G}(\mathbf{c}), I_{B}(\mathbf{c})\right]^{T}$ or $\quad \mathbf{I}(\mathbf{c})=\left[I_{Y}(\mathbf{c}), I_{C b}(\mathbf{c}), I_{C r}(\mathbf{c})\right]^{T}$ is used to represent the RGB (Red, Green and Blue) or $\mathrm{YcbCr}$ (Luminance(Y), Chrominance blue $(\mathrm{Cb})$ and Chrominance red(Cr)) pixel values if it is a color video.

First the composite video noise was acquired through a forward and a reverse conversion process. The forward process converted reference digital component signals [6] to analogue component signals then to composite signals. These composite signals were recorded onto S-VHS tapes. For the reverse process, recorded video signals were first converted from composite signals to analogue component and then to digital signals. The actual composite video noise was then acquired by taking the difference between reference digital video signals and re-digitised video signals. . The composite noise captured by above process can be expressed as follows,

$\eta(\mathbf{c})=z(\mathbf{c})-I(\mathbf{c}), \mathbf{c} \in V$

where $z(\mathbf{c})$ is the degraded(observed) signal; $I(\mathbf{c})$ is the true/reference signal; $\eta(\mathbf{c})$ is denoted as the composite noise/artefacts. Nine standard test VQEG sequences (Water fall, Football, Ship of the line, Suzie, Studio set, Three, Mobile and calender, Animals and Harp)[7] were used to generate a set of composite video noise data. These sequences are either of 625 (component 4:2:2 interlace PAL @ $50 \mathrm{~Hz}$ ) or 525 (component 4:2:2 interlace NTSC @ 50 Hz) format with 220 frames each. Evidently, the noise is visibly structured with a substantial portion of its energy concentrated around vertical and diagonal edges (see Figs. 1f -1o).

\subsection{Statistical properties of the composite noise}

Since exact mathematical/physical models may not be practical, a statistical approach needs to be adopted for this noise modeling. The statistical approach should be a probability model that can capture the noise variability and are computationally tractable. In this paper, the technologies of statistics (probability) models are used to observe and explain patterns exhibited by the composite video noise to build the model.

For the purpose of statistical modeling, a video/an image is treated as a realization of a spatial/tempo-spatial stochastic process defined on some domain. The domain is assumed to be an image or a sequence of frames. A common assumption in video/image modeling is that the underlying video/image process is stationary, i.e. video/image probabilities are invariant to translations in the video sequences /image plane.

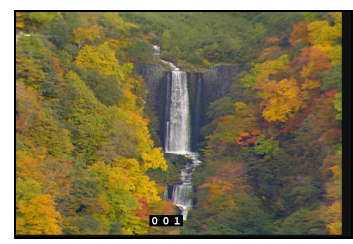

a)

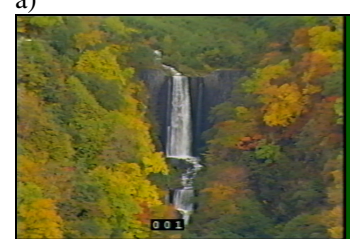

f)

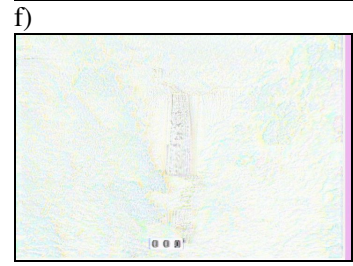

k) 1)

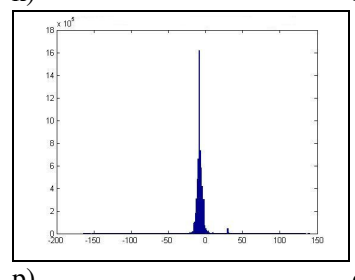

p)
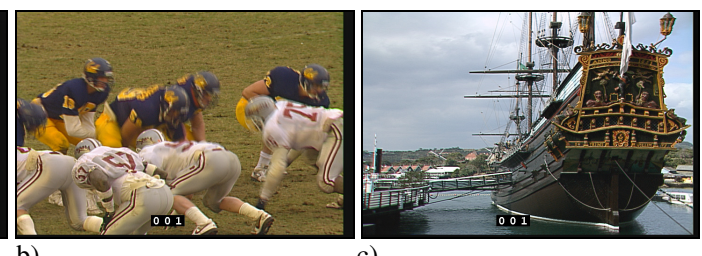

c)
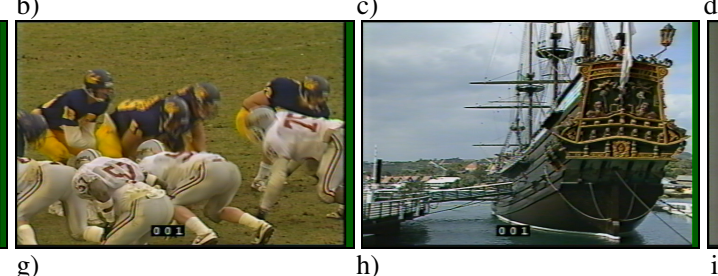

d)
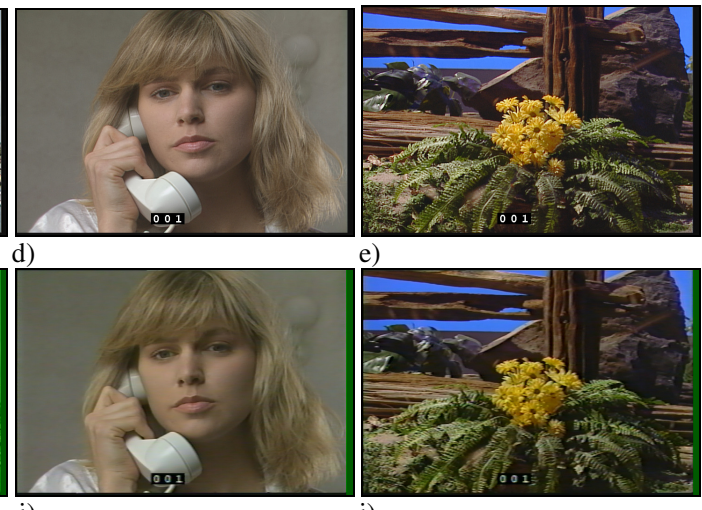

g)

h)

i)
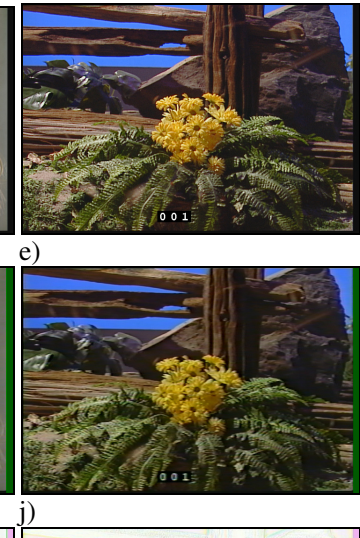

e)

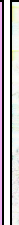

n)

(e) 01

m)
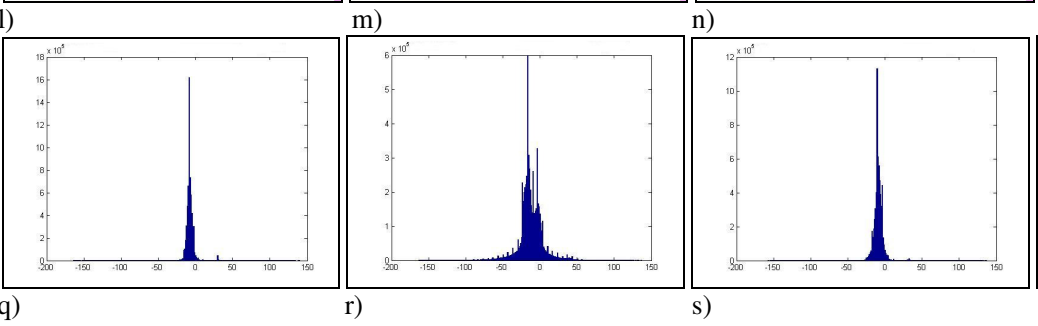

o)

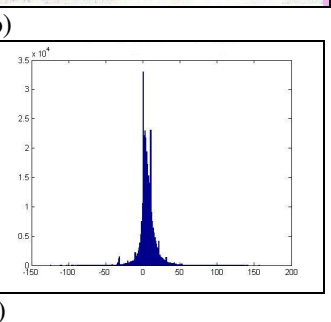



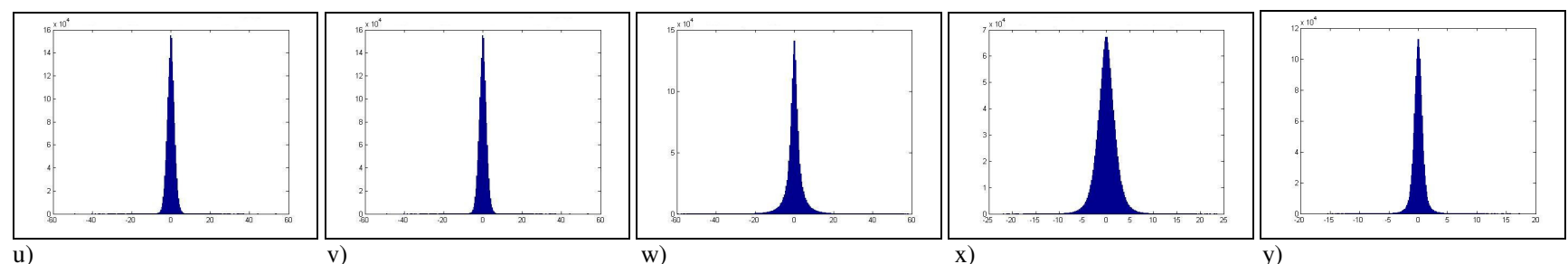

Fig.1. The test video sequences and their processing/decomposition show some composite video noise properties. a) a frame of original test sequence Water fall, b) a frame of original test sequence Foot ball, c) a frame of original test sequence Ship on the line, d) a frame of original test sequence Suzie, e) a frame of original test sequence Studio set. f)-j) the corresponding frames of test sequences shown in Figs.1a-1e degraded by composite noise. k)-o) the absolute difference between the original sequences and their corresponding composite noise degraded sequences shown by the frames from the sequences. p)-t) the histograms of difference between the original sequences and their corresponding composite noise degraded sequences. $u$ )-y) the histograms of the first band wavelet decomposition coefficients difference between the original sequences and their corresponding composite noise degraded sequences for Y component at diagonal orientation.

The histograms of Figs. 1p-1y(bottom two rows) were generated by 30 frames randomly selected from their sequences. Figs. 1p-1t show that the statistical distributions (histograms of composite noise acquired by above mentioned process (1)) vary across the test sequences and there is no this stationary for the direct composite noise statistical information from the sequences. The statistical distributions are dependent on the contents of the video sequences. Recently wavelet based approaches have been recognized as powerful tools for video analysis and denoising. In order to resolve the above problem to model the composite noise, in this paper the Daubechies discrete wavelet [9] is used to decompose the observed and reference video sequences simultaneously in space and frequency for the composite noise modeling.

After the video sequences were decomposed into multi-scale representation and the results of the decomposition were examined (see Figs. 1u -1y), we found that decomposing composite noise using wavelet transforms leads to coefficients that are no-Gaussian distributions, i.e. the distributions/histograms of wavelet coefficients display heavy tails, sharp cusps at the middle (zero) and some extent correlations across different scales. These statistical properties also presented in the coefficients from all above mentioned test sequences decomposed by wavelet. The Fig.2 demonstrates an example of them, the histograms of sequence Harp by a wavelet transformation over three scales and three orientations using Daubechies wavelet bases.

These observations suggests that based on the characters of the coefficients, the statistical composite noise model could be generated, because it seems that the coefficients show common statistical distribution over the test sequences, e.g. a sharp cusp at zero and long exponential tails. Most of them also show symmetric shape if some very small part of their energy is ignored.

\subsection{Model of distribution of wavelet coefficients for composite noise}

We want to represent statistical nature of the wavelet coefficients for composite video noise by means of parametric densities, using only limited parameters. The Fig. 2 shows that coefficients of some scales, orientations and components, of the wavelet decomposition of the composite noise could be described by Gaussian or Laplacian density. However, in order to represent/cover all variability of the distributions for variety of video sequences, we investigate a parametric density, i.e. Generalized Gaussian Model (GGM) that seems to capture the variability of composite noise in video sequences better as it does for natural images. For GGM, marginal densities of natural image coefficients are well modeled [8]. The statistical motivation for using this model come from the shape of the marginals which assume that the coefficient within a subband are independent and identically distributed for the noise(see Figs. 2 and 3); with this assumption, the model could be built by the marginal statistics of the coefficients. These distributions/histograms are well described by a three parameters generalized Gaussian distribution:

$\mathrm{P}_{X}(x ; \mu, \sigma, \rho)=A e^{-[B \mid x-\mu]^{\rho}}, x \in \Re$

where $A=\frac{b \rho}{2 \Gamma(1 / \rho)}, b=\frac{1}{\sigma} \sqrt{\frac{\Gamma(3 / \rho)}{\Gamma(1 / \rho)}}$ and $\Gamma(\cdot)$ is a gamma

function: $\quad \Gamma(x)=\int_{0}^{\infty} t^{x-1} e^{-t} d t, x>0 ; \quad \rho \quad$ is a shape parameter of this distribution: $\rho=1$ corresponds to a Laplacian density and $\rho=2$ corresponds to a Gaussian density; $\mu$ and $\sigma$ are mean and standard deviation. Generally speaking, smaller values of $\rho$ make a density that has more expansive tails and is more concentrated at zero. 

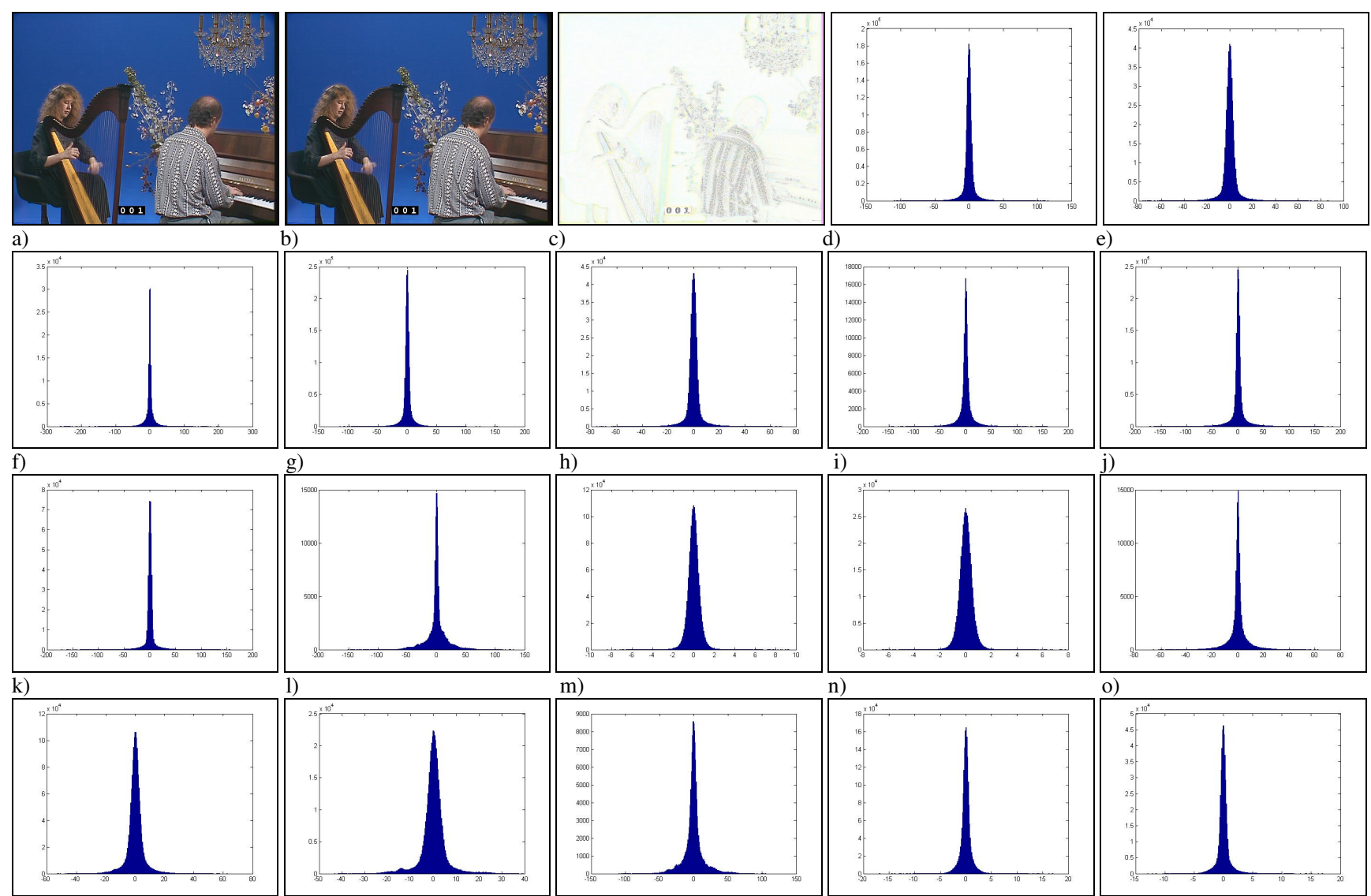

o)
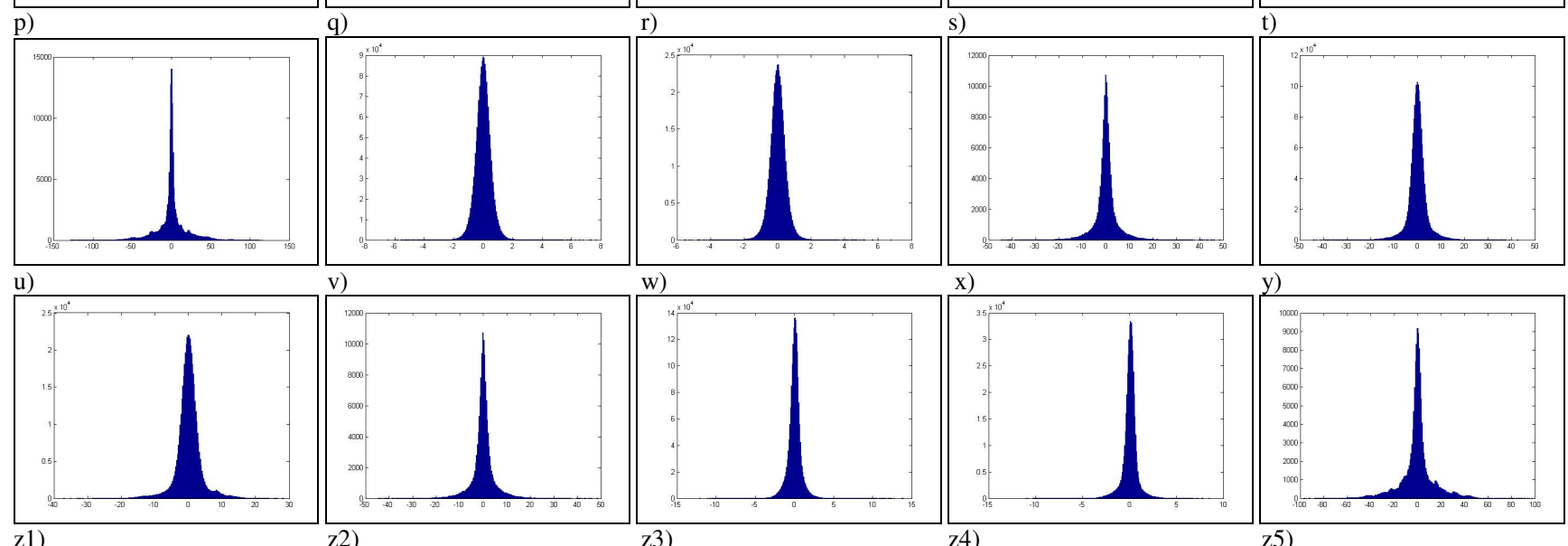

Fig. 2 Histograms of wavelets coefficients, which represent composite noise for each of $\mathrm{Y}, \mathrm{Cb}$ and $\mathrm{Cr}$ components, and their vertical, horizontal and diagonal orientations over three scales for the test video sequence, Harp by random selected 30 frames, a) a fame of the reference sequence, b) the corresponding composite noise degraded frame. c) absolute difference of the two fames. d)-1) the histograms for Y component cross scale 1, 2 and 3, at diagonal, horizontal and vertical orientations, respectively. m)-u) the histograms for $\mathrm{Cb}$ component cross scale 1, 2 and 3, at diagonal, horizontal and vertical orientations, respectively. v)-z5) the histograms for $\mathrm{Cr}$ component cross scale 1,2 and 3, at diagonal, horizontal and vertical orientations, respectively.

The parameters, $\mu, \sigma$ and $\rho$, can be estimated for each subband of the sequences using maximum likelihood. Another way of estimating them is via the (linear) regression of $\log (\log (h(x)+h(-x))-2 \log (h(0)))$ versus $\log (|x|)$, where $h(x)$ is the histogram value at $x$ and $x$ is the variable for bin centers. In order to judge the performance of the proposed probability model, the metrics, which is the Kolmogorov-Smirnov distance, can be used to compare the observed frequencies to the frequencies predicted by the model, on the space of probability distributions.

\section{Composite noise suppression based on the proposed noise model}

After the noise analysis and the stochastic model of the noise wavelet coefficients is represented by empirical modeling of the marginal distributions of wavelet 
coefficients as a subband-dependent mixture of independent, parameterized generalized Gaussian distributions (2), in this section, we discuss how to apply it for suppressing the noise, especially in surveillance videos, because there are still a huge amount of composite surveillance video in archive needed to be enhanced for processing. The proposed method attempts to remove the noise and estimate the original video signal by $F=W T W^{-1}$, where $W$ an $W^{l}$ stand for the forward and inverse discrete wavelet transform, respectively, and the $T$ is a wavelet domain point-wise function which operates on the wavelet coefficients. To improve denoising performance under the wavelet-based denoising framework, a critical task is to seek a better modification strategies/function, $T$ for wavelet coefficients.

Let us denote the noise-free video coefficients as a vector $\vec{I}$ and the coefficients of the noise degraded (observed) video as $\vec{z}$ for all wavelet transform scales and orientations for each component. Our approach is motivated by the property of the modeling: the marginal distributions of wavelet coefficients of the composite noise in a subband are always well approximated by a zero mean Generalized Gaussian probability distribution function (p.d.f.).

The proposed approach for estimation of the noise $\vec{\sigma}_{n}^{2}$, is to use static background/temporal motionless part of surveillance video to conduct this estimation. First the video background or temporal motionless part of the video is segmented by a modified Mixture of $K$ Gaussian background modelling approach [10] or other video motion segmentation techniques. Once the video background is segmented, the $V$ is divided into background/ temporal motionless part, $V_{b}$ and foreground/motion part, $V_{f}$.

Since zero mean is assumed for the Generalised Gaussian p.d.f in (2), a good estimate of the true background pixel value, $\vec{I}(\mathbf{c})$, can be obtained by the pixel-wise average of a large enough number of $N$ (recommended up to 20 frames if available) of the observations $\vec{z}_{n}(\mathbf{c})$, for $n=1, \ldots, N$, as follows.

$\hat{\vec{I}}(\mathbf{c})=\frac{1}{N} \sum_{n=1}^{N} \vec{z}_{n}(\mathbf{c}), \quad \mathbf{c} \in V_{b}$,

The estimate of the parameter $\vec{\sigma}$ is calculated as the unbiased sample standard-deviation on

$$
\hat{\vec{\sigma}}(\mathbf{c})=\sqrt{\frac{1}{N-1} \sum_{n=0}^{N}\left(\vec{z}_{n}(\mathbf{c})-\hat{\vec{I}}(\mathbf{c})\right)^{2}}, \quad \mathbf{c} \in V_{b}
$$

The estimates $\hat{\vec{I}}$ and $\vec{\sigma}$ are distributed, respectively, following a normal distribution and a scaled non-central chi-distribution, which can also be approximated, very accurately for a large $N$, as a normal distribution [11]. After the temporal average based soothing, the background/ motionless part $V_{b}$ are recovered. The method of suppressing the noise for foreground/ motion part $V_{f}$ is introduced as follows,

Under an orthonormal wavelet transformation, based on the minimum mean square estimate $\vec{I}(\mathbf{c})$ by $\hat{\vec{I}}(\mathbf{c})$, the proposed denoising method is a local spatio-temporal image structure and noise property adaptive based pixelwise method, which can express as a local Wiener-like estimate:

$$
\hat{\vec{I}}(\mathbf{c})=K(\mathbf{c}) \vec{z}(\mathbf{c}), \mathbf{c} \in V_{f}
$$

where

$$
K(\mathbf{c})=\frac{\vec{\sigma}^{2}(\mathbf{c})}{\vec{\sigma}^{2}(\mathbf{c})+\vec{\sigma}_{n}^{2}}
$$

where $\vec{\sigma}_{n}^{2}$ is the variance of the composite noise for the coefficients and $\vec{\sigma}^{2}$ is used to represent the structure of the original images. Note that the $\vec{\sigma}_{n}^{2}$ varies cross different subbans even with a frame/video(see Fig. 2).

It means that $K(\mathbf{c}) \leq 1$, and $\vec{I}(\mathbf{c})$ is determined by $K(\mathbf{c})$. Our solution in (5) is that the devised pixel-wise based denoising function is adaptive to noise statistical coefficient distribution parameters for different band levels and image structure around the pixel in order to preserve the fine structure of the video by over smoothing.

Approximately a smooth area of the video is with smaller variance $\vec{\sigma}^{2}$ and the noise level of the video could be represented by its noise variance $\vec{\sigma}_{n}^{2}$, which depends on contents of the frame and level of the wavelet decomposition (see Fig. 2). The proposed denoising approach is devised as that in the smooth or low noise level area of the frame, the output value of $K(\mathbf{c})$ is to be smaller. For example, if $\vec{\sigma}_{n}^{2}=0$ (without noise), $K(\mathbf{c})=1$, i.e. $\hat{\vec{I}}(\mathbf{c})=\vec{z}(\mathbf{c})$, calculated by (5) and (6).

Therefore if $\vec{\sigma}^{2}$ and $\vec{\sigma}_{n}^{2}$ are estimated then the $\hat{\vec{I}}(\mathbf{c})$ will be determined by (5). The proposed approach for the estimation of $\vec{\sigma}_{n}^{2}$, is to use static background/temporal motionless part of the same frame of the surveillance video to conduct this estimation, which introduced in (3) and (4).The estimation of local variance $\vec{\sigma}^{2}$ is conducted as follows. To take full advantage of the strong spatiotemporal correlations of neighboring frames, and a block matching(TSS)[11 8] for foregrounds, based on their motion compensation from a phase-cross based global motion estimation, is applied to adjacent frames, which increases their temporal correlations. Then, the proposed denoising technique for determining the $\vec{\sigma}^{2}$ is to build up an estimate for it by an observation of the wavelet coefficients in a spatio-temporal neighbourhood surrounding $\vec{z}$ (c). The estimation of the variance of each coefficient can be obtained by 


$$
\hat{\vec{\sigma}}^{2}=1 / M \sum_{\mathbf{c} \in N(\mathbf{c})}(\vec{z}(\mathbf{c})-\overline{\bar{z}}(\mathbf{c}))^{2}, \mathbf{c} \in V_{f}
$$

where $N(\mathbf{c})$ is a multi-frames of a 3-D spatio-temporal neighborhood of $\vec{z}_{n}(\mathbf{c})$, which is made by $3 \times 3$ pixel window centered at $\mathbf{c}$ for the wavelet coefficients of current frame $n$, previous frames $n-2$ and $n-1$, and next frames $n+1$ and $n+2$.

After the required $\vec{\sigma}_{n}^{2}$ and $\vec{\sigma}^{2}$ are estimated, in the proposed denoising techniques, the true value of $\hat{\vec{I}}(\mathbf{c})$ is estimated by (5) if $\mathbf{c} \in V_{f}$.

\section{Experiments}

The proposed composite noise modeling and suppression technique were evaluated by a variety of tests video sequences, which including Animals, Tree, Ship on the line, Mobile and calender, Suzie and Studio set. These sequences, with 220 frames each, contain various complicated contents and motions.

\subsection{Parameters estimation for the noise model}

In Fig. 3, the red lines represent the real normalised histograms of the wavelet coefficients of the test sequences, which show the p.d.f. of the coefficient composite noise from the test sequences; the red dash line represent the proposed coefficients composite noise models for the sequences. The parameters of the models were obtained by the maximum likelihood method. The blue lines represent the Gaussian distributions with parameters driven from the wavelet coefficients to try to fit the real p.d.f.. The Fig. 3 demonstrates that the proposed models with the estimated parameters are well fitted to the real p.d.f., because it has a special shape

control parameter $p$, which is 0.87 and 1.12 for the models in Fig. $3 \mathrm{a}$ and Fig. $3 \mathrm{~b}$ respectively. In most cases, the Gaussian distribution can not fit the real p.d.f. well.

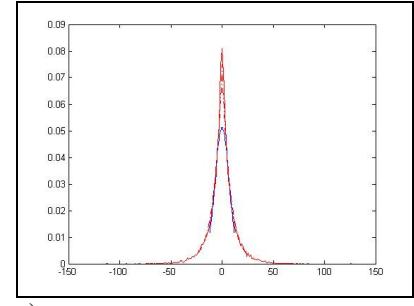

a)

Fig. 3 Normalized histograms of wavelet coefficients for the test sequences Mobile and calender and Studio set, are fitted by the proposed GGM generated from the wavelet coefficients .a) test sequence Mobile and calender, b) test sequence Studio set.

\subsection{Composite noise suppression}

The proposed composite noise suppression technique were evaluated by a range of tests and its performance is compared with some benchmark filtering technique [12] in the area of removing noise in color images. Several objective criteria are used in the tests to measure the restotion in restored images. The objective criteria include the Mean Square Error (MSE) and the Mean Absolute Error (MAE) defined in the RGB color space [3], and the Normalized Color Difference (NCD) [3] which measures the color distortion in the perceptually uniform CIELAB color space.

Four levels of wavelet transformation and their vertical, horizontal and diagonal orientations for each of $\mathrm{Y}, \mathrm{Cb}$ and $\mathrm{Cr}$ components were used in the proposed denoising technique to improve the composite noise degraded test sequences. We verified the proposed technique using the typical test video sequences Tree, Animals and Ship on the line. The selected video sequences represent different types of image structures.

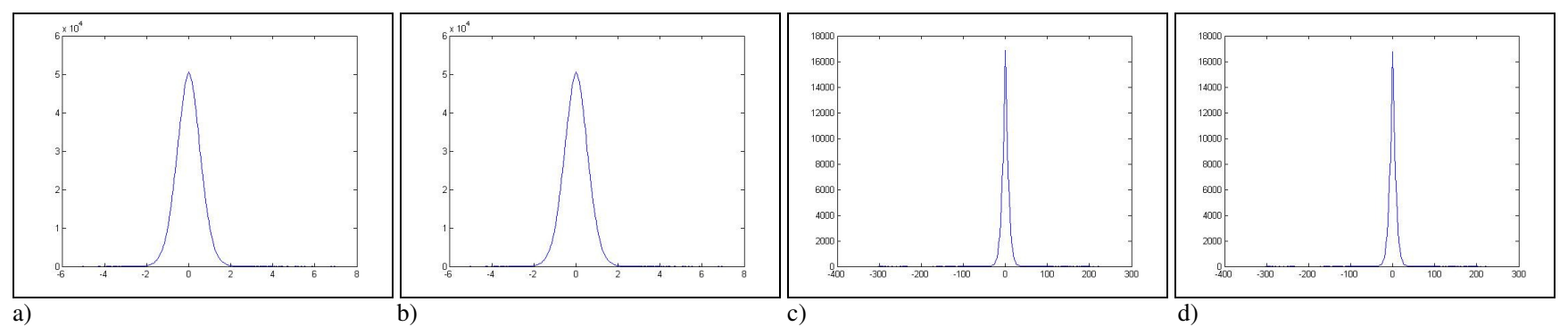

Fig. 4 The composite noise distributions(histograms) driven from the differences based on recovered coefficients by estimated parameters of the proposed model or reference true sequences. a) and b) for test sequence Suzie at scale 2, diagonal orientation and Y component, c) and d) for test sequence Animals at scale 3, horizontal orientation and $\mathrm{Cr}$ component.

The Fig. 4 shows the verification results of parameters estimated from the background or temporal motionless part of degraded sequences. The histograms in Figs. $4 \mathrm{~b}$ and $4 \mathrm{~d}$ were generated by difference between the estimated $\hat{\vec{I}}$ (c) by (3) in background/ temporal motionless part of degraded sequences and the degraded sequences.
The histograms in Figs. 4a and $4 \mathrm{c}$ were generated by degraded sequences and noise-free reference sequences. It can be seen there is not significant difference between the estimated ones and real ones, so the estimated parameters of foreground by (3) and (4) are reliable. 
Table1. Performance of the proposed composite noise supression technique for sequences, Tree, Ship on the line and Animals.

\begin{tabular}{|c|c|c|c|c|c|c|c|c|c|}
\hline \multirow{2}{*}{ Filters } & \multicolumn{3}{|l|}{ Tree } & \multicolumn{3}{|c|}{ Ship on the line } & \multicolumn{3}{|c|}{ Animals } \\
\hline & MSE & MAE & NCD & MSE & MAE & NCD & MSE & MAE & $\mathrm{NCD}$ \\
\hline Degraded & 234.72 & 7.64 & 2.19 & 256.3 & 7.8 & 2.01 & 85.76 & 3.45 & 2.61 \\
\hline Proposed & 202.92 & 6.32 & 1.63 & 235.5 & 7.1 & 1.58 & 74.25 & 3.24 & 2.25 \\
\hline Soft_thresholding[12] & 328.92 & 7.63 & 2.16 & 256.1 & 8.1 & 2.01 & 86.69 & 3.51 & 2.64 \\
\hline
\end{tabular}

The performances for the proposed denoising technique and a compared technique are shown in Table. 1. It can be seen that the proposed denoising technique performs well due to the output of the proposed wavelet based denoising function adapted to signal content and the composite noise level in different bands in the proposed filter. The proposed new spatio-temporal based denoising technique achieves significant enhancement for the degraded sequences in terms of the objective measurements MSE, MAE and NCD while the soft thresholding based approach looks not suit for suppressing composite noise.

\section{Conclusion}

Modelling of pixel level, image level and video level noise characteristics of videos, especially the 3-D spatiotemporal feature based modelling and estimation of the parameters of the model, lays ground work for further video enhancement/noise suppression algorithms. The contributions of the proposed composite noise/artefacts modelling and suppression techniques are that in order to analyse the properties of the composite video noise, based on wavelet decomposition of the noise, subbanddependent mixture of independent, parameterized generalized Gaussian distributions was proposed to model the marginal distributions of wavelet coefficients. Based on the parameters of estimation from the proposed statistical model, a new spatio-temporal based denoising approach was proposed especially to suppress the composite noise in surveillance video. Extensive experiments conducted using standard test video sequences have shown that the proposed composite noise modelling and suppression techniques obtained promising results. The proposed model and estimations will provide a better understanding of composite video noises and artefacts. It facilitates the further design of adaptive filtering, optimisation criteria/rules, and algorithms, based on the model and its parameters estimation, to efficiently suppress the composite noise.

\section{Acknowledgement}

This work was supported by an Australia Research Council Discovery Project grant (DP0988654).

\section{References}

[1] SMPTE, Television - Composite Analog Video Signal NTSC for Studio Applications, SMPTE 170M-2004, (Society of Motion Picture and Television Engineers, Jan. 2004).

[2] A. K. Jain, Fundamentals of Digital Image Processing (Prentice Hall, Inc., Englewood Cliff, NJ, 1989).

[3] R. C. Gonzalez, Digital Image Processing (Prentice Hall, Inc., Englewoord Cliff, NJ, 2nd edition, 2002).

[4] Z. Ma, H. R. Wu, and D. Feng, Partition Based Vector Filtering Technique for Suppression of Noise in Digital Color Images, IEEE Transactions on Image Processing, 15(8), Aug. 2006, 2324-2342.

[5] Z. Xu, H. R. Wu, B. Qiu, and X. Yu, Geometric Features Based Filtering for Suppression of Impulse Noise in Color Images, IEEE Transactions on Image Processing, 18(8), Aug. 2009, 1743-1759.

[6] ITU-R, Studio Encoding Parameters of Digital Television for Standard 4:3 and Wide Screen 16:9 Aspect Ratio, Rec. (BT.6016, ITU-R, Jan. 2007).

[7] http://www.its.bldrdoc.gov/vqeg.

[8] P. Moulin and J. Liu, Analysis of multiresolution image denoising schemes using a generalized Gaussian and complexity priors, IEEE Trans. Info. Theory, 45(3), April, 1999, 909-919.

[9] C. Stauffer \& W.E.L. Grimson, Adaptive background mixture models for real-time tracking, Proc. of CVPR, 1999, 246-252.

[10] I. Daubechies, Ten Lectures on Wavelets (Society for Industrial Mathematics, 1992).

[11] A. Foi, M. Trimeche, V. Katkovnik, and K. Egiazarian, Practical Poissonian-Gaussian Noise Modeling and Fitting for Single-Image Raw-Data, IEEE Transactions on Image Processing, 17(10), Oct. 2008, 1337-1754.

[12] S. G. Chang, B. Yu, and M. Vetterli, Adaptive wavelet thresholding for image denoising and compression, IEEE Trans. Image Process., 9(12), Dec., 2000, 1532-1546.

[13] A. Bolvik, Handbook of Image and Video Processing (Elsevier 2006). 